\title{
ON THE TIME AND CELL DEPENDENCE OF THE COARSE-GRAINED ENTROPY. II
}

\author{
P. HOYNINGEN-HUENE \\ Institut für Theoretische Physik der Universität Zürich, \\ CH-8001 Zürich, Switzerland
}

Received 21 October 1975

\begin{abstract}
We present calculations of the coarse-grained entropy $S_{\mathrm{cg}}(t)$ for the model of a classical point particle enclosed in a two-dimensional box with perfectly reflecting walls. Wc find in comparison with the one-dimensional case that the fluctuations of $S_{\mathrm{cg}}(t)$ and of the expectation values of the position have decreased, and that $S_{\mathrm{cg}}(t)$ does not take its asymptotic value at regular time intervals. The times after which $S_{\mathrm{cs}}(t)$ and the expectation values have approximately reached their equilibrium values, are about equal; recurrence times are rather clearly separated from this time. Finally, we give a general estimate on the change of an expectation valuc due to a refinement of the cells.
\end{abstract}

\section{Introduction}

In a previous paper ${ }^{1}$ ) (referred to as I), we have discussed properties of the coarse-grained entropy in general, and we have calculated the coarse-grained entropy $S_{\mathrm{cg}}(t)$ for a simple model system, namely a classical free particle in a onedimensional box with perfectly reflecting walls ("PIB-1 model"). Initially, the particle is confined to a certain part of the box; this constraint is released at $t=0$. The main results of those model calculations were:

a) $S_{c g}(t)$ approaches its new equilibrium value nonmonotonically;

b) $S_{\mathrm{cg}}(t)$ depends on the number of cells very weakly from remarkably few cells on;

c) $S_{\mathrm{cg}}(t)$ approaches its equilibrium value faster (essentially $\sim 1 / t^{2}$ ) than the expectation value of the position of the particle $\langle q\rangle(t)$ (essentially $\sim 1 / t$ );

d) The stability region where the expected relaxation time $\left.{ }^{1}\right)\left\langle\tau_{\text {rel }}\right\rangle$ is fairly stable against an increase of the number of cells, is very small, since the fluctuations of $\langle q\rangle(t)$ around the new equilibrium value are large.

The limitations of the PIB-1 model are evident: firstly the low number of dimensions of phase space $\Gamma(\operatorname{dim} . \Gamma=2$ ), and secondly the highly singular interaction with the walls of the box (perfect reflection). The first defect seems even more 
serious, since for one particle in one dimension, one cannot expect a clear distinction between a recurrence time and an (expected) relaxation time. A separation of these two time scales is highly desirable, however, since within which time $S_{\mathrm{cg}}(t)$ reaches approximately its new equilibrium value, is an important question.

In this paper, we shall therefore present calculations of $S_{\mathrm{cg}}(t)$ for a particle in a two-dimensional box with perfectly reflecting walls ("PIB-2 model"). As we shall see, some of the unpleasant properties of the one-dimensional case will be absent in the two-dimensional model. In section 2, we shall solve the Liouville equation for the model, and calculate various quantities. We shall present the results in section 3; furthermore, estimates on the change of an expectation value due to subpartitions of the phase cells will be given. The paper closes in section 4 with a discussion and summary.

\section{The model}

The system consists of one classical free point particle of mass $m$ enclosed in a two-dimensional rectangular box with perfectly reflecting walls of length $L$ and $x L, 0<\alpha \leqslant 1$. We describe the particle in an energy "shell" $\Sigma$, consisting of all points $\left(q_{1}, q_{2}, p_{1}, p_{2}\right) \in \mathscr{R}^{4}$ with

$$
0 \leqslant q_{1} \leqslant L, \quad 0 \leqslant q_{2} \leqslant \alpha L, \quad\left(p_{1}^{2}+p_{2}^{2}\right) / 2 m \leqslant E,
$$

where $E$ is the maximum energy of the particle. With the invariant measure $\mathrm{d} \mu$ $=\mathrm{d} q_{1} \mathrm{~d} q_{2} \mathrm{~d} p_{1} \mathrm{~d} p_{2}$ we have

$$
\mu(\Sigma)=2 \pi m E \alpha L^{2} \text {. }
$$

For $t \leqslant 0$, we shall have an equilibrium state where the particle has arbitrary energy between zero and $E$, and is confined to that part of the box with $0 \leqslant q_{1}$ $\leqslant q_{1}^{\max }$ and $0 \leqslant q_{2} \leqslant q_{2}^{\max }$, where $0<q_{1}^{\max }<L$ and $0<q_{2}^{\max }<\alpha L$. We thus get for the distribution function

$$
\varrho\left(q_{1}, q_{2}, p_{1}, p_{2} ; t \leqslant 0\right)=\left[\begin{array}{l}
\left(2 \pi m E q_{1}^{\max } q_{2}^{\max }\right)^{-1} \\
\text { for } 0 \leqslant q_{1} \leqslant q_{1}^{\max } \\
0 \text { elsewhere }
\end{array} \text { and } 0 \leqslant q_{2} \leqslant q_{2}^{\max }\right.
$$

which has been normalized to unity. Imposing reflecting boundary conditions for $t \geqslant 0$ we obtain as initial condition

$$
\begin{aligned}
& \varrho\left(q_{1}, q_{2}, p_{1}, p_{2} ; t=0\right) \\
& =c_{1} \sum_{i_{1} \in \mathscr{Y}} \theta\left(q_{1}+q_{1}^{\max }-2 i_{1} L\right) \theta\left(q_{1}^{\max }+2 i_{1} L-q_{1}\right) \\
& \quad \times \sum_{i_{2} \in \mathscr{Y}} \theta\left(q_{2}+q_{2}^{\max }-2 i_{2} \alpha L\right) \theta\left(q_{2}^{\max }+2 i_{2} \alpha L-q_{2}\right)
\end{aligned}
$$


with

$$
c_{1}=\left(2 \pi m E q_{1}^{\max } q_{2}^{\max }\right)^{-1}
$$

Since the hamiltonian consists only of the kinetic part, we get the solution of the Liouville equation for $t \geqslant 0$ with the initial condition (2.4) by replacing $q_{i}$ by $q_{i}-p_{i} t / m$ in $\left.(2.4)^{2}\right)$. This yields

$$
\begin{aligned}
& \varrho\left(q_{1}, q_{2}, p_{1}, p_{2} ; t\right) \\
& =c_{1} \sum_{i_{1} \in X} \theta\left(q_{1} / L-p_{1} t / m L+q_{1}^{\max } / L-2 i_{1}\right) \\
& \quad \times \theta\left(q_{1}^{\max } / L+2 i_{1}-q_{1} / L+p_{1} t / m L\right) \\
& \quad \times \sum_{i_{2} \in Z^{2}} \theta\left(q_{2} / \alpha L-p_{2} t / m \alpha L+q_{2}^{\max } / \alpha L-2 i_{2}\right) \\
& \quad \times \theta\left(q_{2}^{\max } / \alpha L+2 i_{2}-q_{2} \mid \alpha L+p_{2} t / m \alpha L\right) .
\end{aligned}
$$

As in PIB-1, @ converges weakly ${ }^{3}$ ) to the microcanonical distribution function in $\Sigma$, that is

$$
\varrho_{\text {eq }}=\left(4 \alpha L^{2}\right)^{-1} \int_{0}^{L} \mathrm{~d} q_{1} \int_{0}^{\alpha L} \mathrm{~d} q_{2} \varrho\left(q_{1}, q_{2}, p_{1}, p_{2} ; 0\right)=[\mu(\Sigma)]^{-1} .
$$

The two assumptions about $\varrho$ in paper I, section 2, are therefore fulfilled for the model.

As in 1 , we take as a macroscopic quantity the position of the particle, thus we have

$$
A_{1}\left(q_{1}, q_{2}, p_{1}, p_{2}\right)=q_{1} \quad \text { and } \quad A_{2}\left(q_{1}, q_{2}, p_{1}, p_{2}\right)=q_{2}
$$

The corresponding accuracies will be $L / j_{1}^{\max }$ for $A_{1}$ and $\alpha L / j_{2}^{\max }$ for $A_{2}$, respectively. This yields the cells ${ }^{4}$ )

$$
\begin{aligned}
\Omega_{j_{1} j_{2}}= & \left\{\left(q_{1}, q_{2}, p_{1}, p_{2}\right) \in \sum \mid\left(j_{1}-1\right) L i j_{1}^{\max } \leqslant q_{1} \leqslant j_{1} L / j_{1}^{\max }\right. \\
& \text { and } \left.\quad\left(j_{2}-1\right) \alpha L i j_{2}^{\max } \leqslant q_{2} \leqslant j_{2} x L / j_{2}^{\max }\right\} \\
& 1 \leqslant j_{1} \leqslant j_{1}^{\max }, \quad \quad \quad 1 \leqslant j_{2} \leqslant j_{2}^{\max }
\end{aligned}
$$

Obviously, we have cells of equal volume

$$
\mu\left(\Omega_{j_{1} j_{2}}\right)=\mu(\Sigma) /\left(j_{2}^{\max } j_{2}^{\max }\right)
$$


For the calculation of the coarse-grained distribution function, we introduce the following dimensionless quantities

$$
\begin{aligned}
& x_{1}:=q_{1} / L, \quad x_{1}^{\max }:=q_{1}^{\max } / L, \\
& x_{2}:=q_{2} /(\alpha L), \quad x_{2}^{\max }:=q_{2}^{\max } /(\alpha L), \\
& y_{1}:=p_{1} t /(m L), \\
& y_{2}:=p_{2} t /(m \alpha L) .
\end{aligned}
$$

This yields

$$
\mathrm{d} \mu=\alpha^{2} L^{4} m^{2} / t^{2} \mathrm{~d} x_{1} \mathrm{~d} x_{2} \mathrm{~d} y_{1} \mathrm{~d} y_{2} .
$$

With the abbreviations

$$
c_{2}(t):=x^{2} L^{4} m^{2} c_{1} / t^{2}
$$

and

$$
R_{t}:=t(2 E / m)^{\frac{1}{2}} / L
$$

we get for the coarse-grained distribution function

$$
\begin{aligned}
& P_{j_{1} j_{2}}(t):=\left(\mu\left(\Omega_{j_{1} j_{2}}\right)\right)^{-1} \int_{2_{j_{1} j_{2}}} \varrho\left(q_{1}, q_{2}, p_{1}, p_{2} ; t\right) \mathrm{d} \mu \\
& =\left(\mu\left(\Omega_{j_{1} j_{2}}\right)\right)^{-1} \mathcal{c}_{2}(t) \sum_{i_{1} \in \mathscr{X}} \sum_{i_{2} \in \mathscr{Y}} \\
& \int_{\left(j_{1}-1\right) / j_{1} \max }^{j_{1} / T^{\max }} \mathrm{d} x_{1} \int_{-R_{t}}^{R_{t}} \mathrm{~d} y_{1} \theta\left(x_{1}-y_{1}+x_{1}^{\max }-2 i_{1}\right) \theta\left(x_{1}^{\max }+2 i_{1}-x_{1}+y_{1}\right) \\
& \times \int_{\left(j_{2}-1\right) / j_{2}{ }^{\max }}^{j_{2} / j_{2} \max } \mathrm{d} x_{2} \int_{-\left(R_{\tau}{ }^{2} y_{1}\right)^{1 / 2 / \alpha}}^{\left(R_{t}{ }^{2}-y_{1}{ }^{2}\right)^{1 / 2 / 2} / x} \mathrm{~d} y_{2} \theta\left(x_{2}-y_{2}+x_{2}^{\max }-2 i_{2}\right) \\
& \times \theta\left(x_{2}^{\max }+2 i_{2}-x_{2}+y_{2}\right) \text {. }
\end{aligned}
$$

The second double integral in (2.15) is a function of $y_{1}$ alone, as far as the integration variables are concerned. We thus define

$$
\begin{aligned}
f\left(y_{1}\right):= & \int_{\left(j_{2}-1\right) / j_{2} \max }^{j_{2} / j_{2} \max } \mathrm{d} x_{2} \int_{-\left(R_{t}{ }^{2}-y_{1}^{2}\right)^{1 / 2 / \alpha}}^{\left(R_{t}^{2}-y_{1}{ }^{2}\right)^{1 / 2} / x} \mathrm{~d} y_{2} \theta\left(x_{2}-y_{2}+x_{2}^{\max }-2 i_{2}\right) \\
& \times \theta\left(x_{2}^{\max }+2 i_{2}-x_{2}+y_{2}\right) .
\end{aligned}
$$


$f\left(y_{1}\right)$ may be calculated exactly, since it is just the area of the intersection of the rectangle defined by the integration boundaries with the strip defined by the $\theta$-functions in the $x_{2}-y_{2}$ plane. The remaining two integrations in (2.15) then integrate $f\left(y_{1}\right)$ over an area of the same structure as that in (2.16) in the $x_{1}-y_{1}$ plane. Since $f$ does not depend on $x_{1}$ we may write for (2.15)

$$
P_{j_{1} j_{2}}(t)=\left[\mu\left(\Omega_{j_{1} j_{2}}\right)\right]^{-1} c_{2}(t) \sum_{i_{1} \in \mathscr{Z}} \sum_{i_{2} \in \mathscr{Z}} \int_{-R_{t}}^{R_{t}} h\left(y_{1}\right) f\left(y_{1}\right) \mathrm{d} y_{1},
$$

where $h\left(y_{1}\right)$ measures the extension of the integration domain in $x_{1}$-direction and may be calculated exactly. We have thus reduced the four-dimensional integration in (2.15) to a one-dimensional one which must be done numerically.

Finally, we note that the sums in (2.15) [and (2.17)] consist only of a finite number of terms. One may show that only those terms contribute inside $\Sigma$ for which the following inequalities hold:

$$
-\left(x_{1}^{\max }+R_{t}\right)<2 i_{1}<\left(1+x_{1}^{\max }+R_{t}\right)
$$

and

$$
-\left(x_{2}^{\max }+R_{t} / \alpha\right)<2 i_{2}<\left(1+x_{2}^{\max }+R_{t} / \alpha\right) .
$$

Having calculated the $P_{j_{1} j_{2}}(t)$ we get for the coarse-grained entropy

$$
S_{\mathrm{ig}}(t)=-k \sum_{j_{1}=1}^{j_{1} \max } \sum_{j_{2}=1}^{j_{\text {max }}} \mu\left(\Omega_{j_{1} j_{2}}\right) P_{j_{1} j_{2}}(t) \ln P_{j_{1}, j_{2}}(t)
$$

according to formula (I.3.9). Following the definition of the expectation value (1.3.8) we calculate

$$
\int_{\Omega_{1} j_{2}} q_{1} \mathrm{~d} \mu=2 \pi m E \alpha L^{3}\left(j_{1}-0.5\right) /\left(\left(j_{1}^{\max }\right)^{2} j_{2}^{\max }\right)
$$

and

$$
\int_{g_{j_{1}} j_{2}} q_{2} \mathrm{~d} \mu=2 \pi m E \alpha^{2} L^{3}\left(j_{2}-0.5\right) /\left(j_{1}^{\max }\left(j_{2}^{\max }\right)^{2}\right)
$$

to be inserted into the formula

$$
\left\langle q_{i}\right\rangle(t)=\sum_{j_{1}=1}^{j_{1} \max } \sum_{j_{2}=1}^{j_{2} \max } P_{j_{1} j_{2}}(t) \int_{g_{j_{1}} j_{2}} q_{i} \mathrm{~d} \mu, \quad i=1,2 .
$$


Now we calculate $P^{(\infty)}\left(q_{1}, q_{2} ; t\right)$ according to (I.4.28). With the substitutions (2.11) we obtain

$$
\begin{aligned}
& P^{(\infty)}\left(q_{1}, q_{2} ; t\right)=\left(4 \pi^{2} E^{2} x_{1}^{\max } x_{2}^{\max } t^{2}\right)^{-1} \sum_{i_{1} \in \mathscr{Y}} \sum_{i_{2} \in Y} \\
& \int_{-R_{t}}^{R_{t}} \mathrm{~d} y_{1} \theta\left(y_{1}-x_{1}+x_{1}^{\max }+2 i_{1}\right) \theta\left(x_{1}+x_{1}^{\max }-2 i_{1}-y_{1}\right) \\
& \quad \int_{-\left(R_{t}^{2}-y_{1}^{2}\right)^{1 / 2} / \alpha}^{\left(R_{t}^{2}-y_{1}^{2}\right)^{1 / 2} / x} \mathrm{~d} y_{2} \theta\left(y_{2}-x_{2}+x_{2}^{\max }+2 i_{2}\right) \theta\left(x_{2}+x_{2}^{\max }-2 i_{2}-y_{2}\right),
\end{aligned}
$$

where the $y_{2}$-integration can be done exactly; in the sum, only those terms contribute, for which (2.18) and (2.19) hold. Inserting the result into (I.4.30) yields $S_{\mathrm{cg}}^{(\infty)}(t)$; the two integrations have to be done numerically.

\section{Results}

For the actual computation we put

$$
L=1, \quad E=1, \quad m=2 \text {; }
$$

this normalizes the time scale so that it takes the particle with maximum momentum $(2 m E)^{\frac{1}{2}}$ the unit time to pass the distance $L$. The accuracy in the numerical integration of (2.17) has been such that the deviation of the norm of $P$ from 1 has been less than 0.7 permille. A typical result for $S_{\mathrm{cg}}(t)$ and $S_{\mathrm{cg}}^{(\infty)}(t)$ (with the parameters $\alpha=\frac{2}{3}, x_{1}^{\max }=\frac{1}{2}, x_{2}^{\max }=\frac{1}{2}$ ) is given in fig. 1 ; the curves are results of interpolation of calculated points of distance $\Delta t=\frac{1}{16}$.

As in the one-dimensional case the approach to the asymptotic value is nonmonotonic, but due to the more complicated geometry of the energy shell, $S_{\mathrm{cg}}(t)$ does not take its asymptotic value in the time interval $[0,3]$. The relative height of the first fluctuation of $S_{\mathrm{cg}}^{(x)}(t)$ (relative to the change of $S_{\mathrm{cg}}^{(\infty)}(t)$ due to the change of state) is here about $1 / 170$, whereas in PIB-1 it has been about $1 / 35$, thus the fluctuation amplitude has decreased. The same effect is seen in the plots of $\left\langle q_{1}\right\rangle(t)$ and $\left\langle q_{2}\right\rangle(t)$ in fig. 2 for the same choice of parameters as in fig. 1 .

The relative height of the first fluctuation has decreased from about $1 / 5.8$ in PIB-1 to $1 / 9.5$ in PIB-2. As seen in fig. 3, this leads to a larger region of stability of the expected relaxation time [see (1.5.23)], since it takes higher accuracy than in the one-dimensional case to detect the fluctuations.

We note here one further general property of the coarse-graining process that has not been included in I which concerns the following requirement. Suppose we change the accuracy of the measurement of a macroscopic quantity. This 

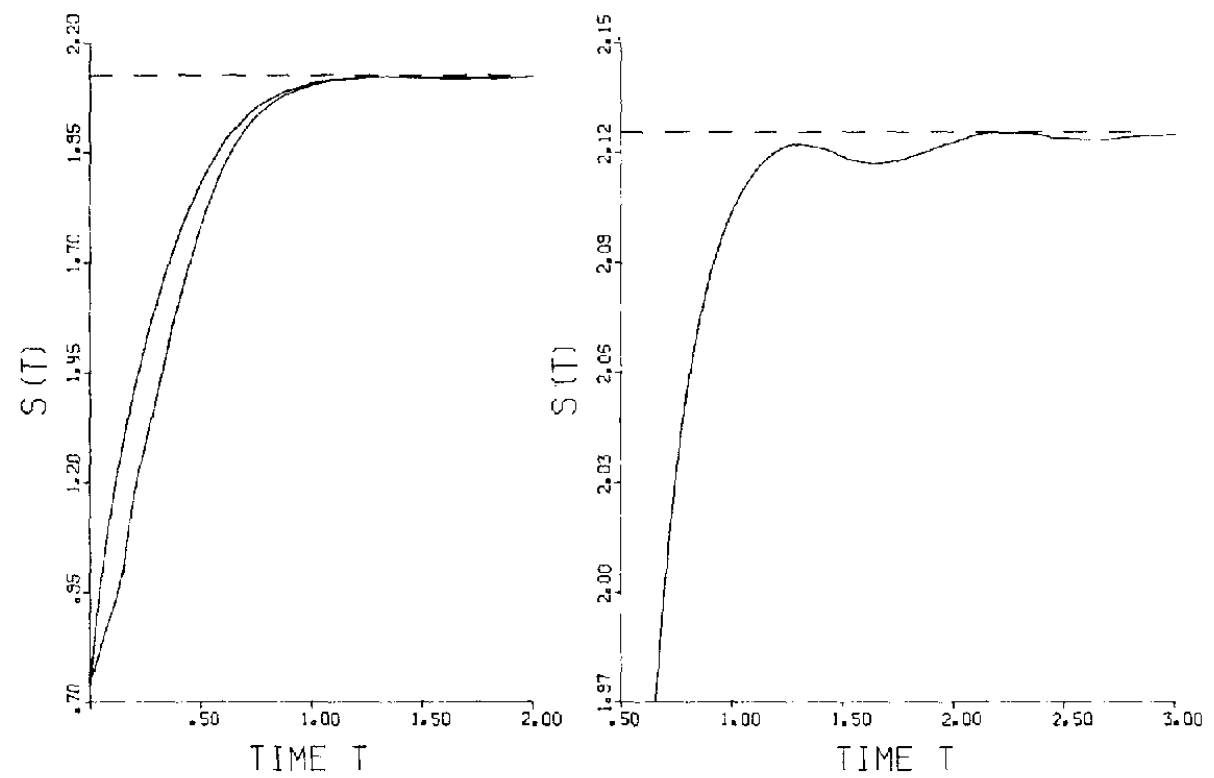

Fig. I. The coarse-grained entropy for $j_{1}^{\max }=j_{2}^{\max }=2$ and $j_{1}^{\max }=j_{2}^{\max }=x$ (below); the other parameters are $\alpha=\frac{2}{3}, x_{1}^{\max }=x_{2}^{\max }=\frac{1}{2}$. In b), the plot for $j_{1}^{\max }=j_{2}^{\max }=2$ has been omitted; it would be just slightly above the given one for infinitely many cells.

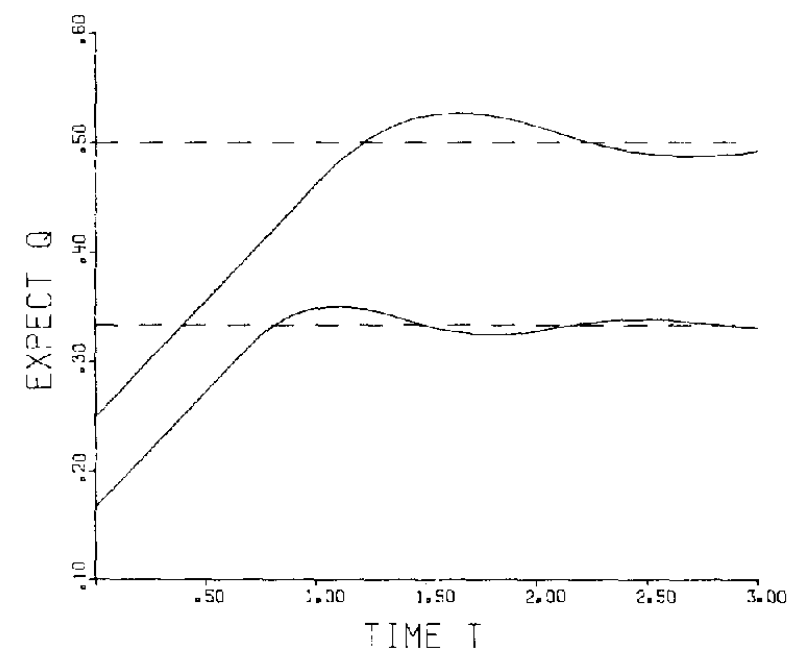

Fig. 2. The expectation values $\left\langle q_{1}\right\rangle(t)$ and $\left\langle q_{2}\right\rangle(t)$ (below) for $j_{1}^{\max }=j_{2}^{\max }=2, \quad x=\frac{2}{3}$, $x_{1}^{\max }=x_{2}^{\max }=\frac{1}{2}$. The dashed lines represent the asymptotic values. 
changes the set of phase cells, and thus the coarse-grained distribution function. One may therefore suspect that the time behavior of the expectation value of another macroscopic quantity is essentially affected by this change which should not be the case. We give now an estimate on the induced change of the expectation values.

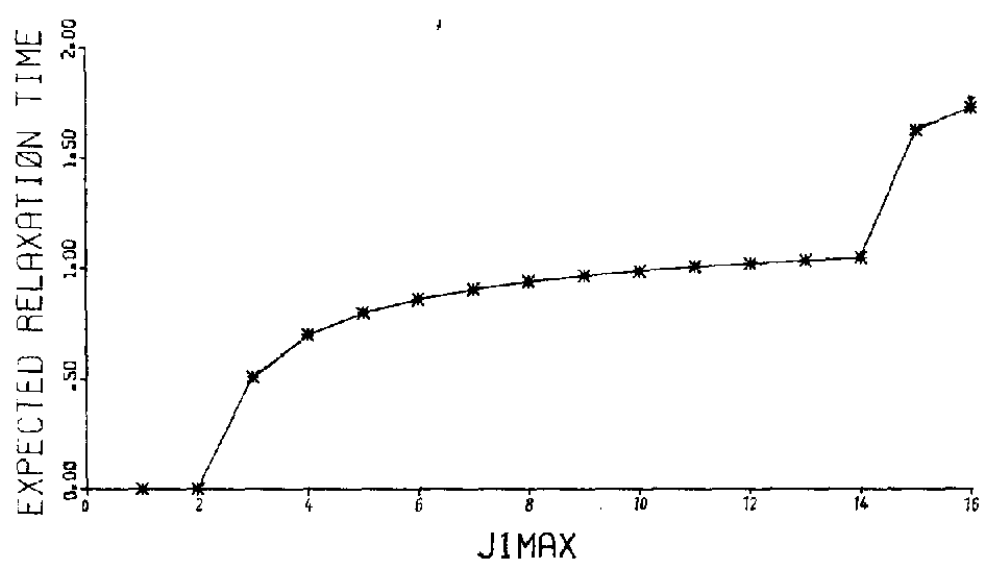

Fig. 3. Expected relaxation time $\left\langle\tau_{\mathrm{rel}}\right\rangle$ as a function of $j_{1}^{\max }$ with $j_{2}^{\max }=2$, other parameters as before. $j_{2}^{\max }=3$ yields the same result, see end of section 3 .

Suppose we have a subpartition $\left\{\Omega_{j}^{m}\right\}$ of $\left\{\Omega_{j}\right\}$ (as in section 4.5 of I), and the subpartition shall be induced by an increase of the accuracy for the measurement of an $A_{k}^{\text {exp }}$. With the original $P(x ; t)$ we get

$$
\left\langle A_{i}\right\rangle(t):=\sum_{j} P_{j}(t) \int_{\Omega_{j}} A_{i}(x) \mathrm{d} \mu=\sum_{j} P_{j}(t) \mu\left(\Omega_{j}\right) A_{i}^{\langle j\rangle},
$$

where $A_{i}^{\langle j\rangle}$ is the mean value of $A_{i}(x)$ in $\Omega_{j}$ with

$$
a_{i}^{\left(v_{j}\right)} \leqslant A_{i}^{\langle j\rangle} \leqslant a_{i}^{\left(v_{j}+1\right)}
$$

according to the definition of the cells (I.3.7). For the refined set of phase cells we get

$$
\overline{\left\langle A_{i}\right\rangle}(t):=\sum_{j, m} P_{j}^{m}(t) \int_{\Omega_{j} m} A_{i}(x) \mathrm{d} \mu=\sum_{j, m} P_{j}^{m}(t) \mu\left(\Omega_{j}^{m}\right) A_{i}^{\langle j m\rangle} .
$$

Now since the $\Omega_{j}^{m}$ are subsets of $\Omega_{j}$ we have again

$$
a_{i}^{\left(v_{j}\right)} \leqslant A_{i}^{\langle j m\rangle} \leqslant a_{i}^{\left(v_{j}+1\right)} .
$$


Putling

$$
\eta_{i}^{j m_{i}}:=A_{i}^{\langle j\rangle}-A_{i}^{\langle j m\rangle}
$$

we get from (3.3) and (3.5)

$$
\left|\eta_{i}^{j m}\right| \leqslant \max _{v_{j}}\left(a_{i}^{\left(v_{j}+1\right)}-a_{i}^{\left(v_{j}\right)}\right)=: \gamma_{i}
$$

This implies

$$
\left\langle A_{i}\right\rangle(t)-\overline{\left\langle A_{i}\right\rangle}(t)|=| \sum_{j, m} P_{j}^{m}(t) \mu\left(\Omega_{j}^{m}\right) \eta_{i}^{j m} \mid \leqslant \eta_{i}
$$

where we have used (3.6), (I.4.16), the normalization of the $P_{j}^{m}(t)$ to unity, and finally (3.7). We have thus the result that the maximum change of an expectation value due to a subpartition of the original cells (introduced by higher accuracy for the same or another macroscopic quantity), is less than its maximum inaccuracy. Noticing that both estimates (3.7) and (3.8) are fairly rough we may expect an even smaller change.

For the PIB 2 model we may even show that a change of $j_{2}^{\text {max }}$ leaves $\left\langle q_{1}\right\rangle(t)$ invariant (and vice versa) which is a consequence of the particularly simple "macroscopic" variables. Defining the coarse-grained distribution function

$$
\left.P\left(q_{1}, q_{2} ; t\right)\right|_{\left(q_{1}, q_{2}\right) \in \Omega_{j_{1} j_{2}}}:=P_{j_{1} j_{2}}(t)
$$

which implies a correspondence of the $q_{i}$ to the $j_{i}$, we have

$$
\left\langle q_{1}\right\rangle=\int_{\Sigma} \mathrm{d} \mu P\left(q_{1}, q_{2} ; t\right) q_{1}=2 \pi m E \int_{0}^{L} \mathrm{~d} q_{1} q_{1} \int_{0}^{x L} \mathrm{~d} q_{2} P\left(q_{1}, q_{2} ; t\right) .
$$

Now we show that the function $\int_{0}^{\alpha L} \mathrm{~d} q_{2} P\left(q_{1}, q_{2}, t\right)$ does not depend on the particular choice of $j_{2}^{\max }$. Calculating

$$
\begin{aligned}
\int_{0}^{\alpha L} P\left(q_{1}, q_{2} ; t\right) \mathrm{d} q_{2} & =\sum_{j_{2}=1}^{j_{2}{ }^{\max }} P_{j_{1} j_{2}}(t) \alpha L / j_{2}^{\max } \\
& =j_{1}^{\max } /(2 \pi m E L) \sum_{j_{2}=1}^{j_{2} \max } P_{j_{1} j_{2}}(t) \mu\left(\Omega_{j_{1} j_{2}}\right)
\end{aligned}
$$

according to (3.9) and (2.10), we see from (I.4.16) that the last sum in (3.11) is independent of the cell structure in $q_{2}$ direction. 


\section{Discussion and summary}

It is instructive to summarize now the main results and differences of PIB-1 and PIB-2.

a) Both models show a very weak dependence of $S_{\mathrm{cg}}(t)$ on the number of cells, especially after a time of the order of the expected relaxation time has elapsed.

b) In PIB-1, $S_{\mathrm{cg}}(t)$ takes its asymptotic value at regular time intervals. This is not the case in PIB-2 (at least not in the time interval considered). Here PIB-1 should be the exception to that general behavior.

c) The fluctuations both of $S_{\mathrm{cg}}(t)$ and of the expectation values around their asymptotic values have decreased in PIB-2 in comparison to PIB-1. This leads to a larger (approximate) plateau in a plot of $\left(\tau_{\mathrm{rc} 1}\right\rangle v s$. the accuracy of the measurement. We expect this trend continues for higher dimensionality of $\Sigma$.

d) Another difference between PIB-1 and PIB-2 concerns the separation of time scales. For accuracies that do not detect the fluctuations, the expected relaxation time is about unity in both models; $S_{\mathrm{cg}}(t)$ has reached its equilibrium value after this time with even better relative accuracy than the expectation values. For a qualitative investigation of the recurrence times take a particle with maximum energy $E$. In PIB-1, the particle will have reached again its precise initial condition after a time $=2$. For the particle with maximum energy $E$ in PIB-2, however, recurrence times vary from $2 \alpha$ (e.g. $\left.\alpha=\frac{2}{3}\right)$ to infinity, depending mainly on the angle between $p_{1}$ and $p_{2}$, and the accuracy within which the particle shall reach its initial condition again. We may therefore expect that an average recurrence time (however the averaging is done) is better separated from the expected relaxation time, than in PIB-1. It is now important to note that in both models the time after which $S_{\mathrm{cg}}(t)$ has approximately reached its equilibrium value, is by far closer to $\left\langle\tau_{\text {rel }}\right\rangle$ than to an (average) recurrence time; because of the better separation of these times in PIB-2, the present results have more weight. Therefore, in the old controversy about the time after which $S_{\mathrm{cg}}(t)$ has reached approximately stationary values ${ }^{5,6,7}$ ), the assertion is supported that this time is much shorter than the recurrence time.

We may conclude that the results presented in this paper give further support to the opinion that the coarse-grained entropy is a proper microscopic expression for the entropy for both equilibrium and nonequilibrium.

\section{Acknowledgements}

I wish to thank A.Thellung for discussions, and M.S.Harle for reading the English manuscript. 


\section{References}

1) P.Hoyningen-Huene, Physica 82A (1976) 417; a preliminary account of this work has been given in Helv. Phys, Acta 48 (1975) 39.

2) I.Prigogine, Nonequilibrium Statistical Mechanics (Interscience, New York, 1962).

3) H.Grad, Comm. Pure Appl. Math. 14 (1961) 323.

4) N.G. van Kampen, in Fundamental Problems in Statistical Mechanics, E.G.D.Cohen, ed. (North-Holland, Amsterdam, 1962).

5) P. and T.Ehrenfest, Encykl, math. Wiss. IV 4 (1911).

6) R.C.Tolman, The Principles of Statistical Mechanics (Oxford Univ. Press, London, 1938).

7) D. ter Haar, Rev. Mod. Phys. 27 (1955) 289. 